\title{
DISTINGUISHED RINGS OF LINEAR TRANSFORMATIONS $\left({ }^{1}\right)$
}

\author{
BY \\ R. E. JOHNSON
}

A ring $R$ of linear transformations of a vector space $M$ over a division ring $D$ is called distinguished iff (1) the lattice $J$ of all $R$-submodules of $M$ is a distributive sublattice of the lattice $L$ of all subspaces of $M$, and (2) the set of all linear transformations of $M$ leaving $J$ invariant is $R$. The study of such rings is motivated by a paper of Wolfson [2] in which $J$ is a chain and by several recent papers of Behrens [4]. Behrens studies Artinian rings $R$ with unity having faithful $R$ modules $M$ such that the lattice of $R$-submodules of $M$ is distributive.

Our primary interest is with distinguished rings $R$ for which $J$ is finite as well as distributive. Such a condition does not force the ring $R$ to be Artinian. A basic tool in our study is a lattice theorem (1.1) stating that every element of $J$ is a direct sum of elements of $L$ associated with the irreducible elements of $J$. It is shown that every finite distributive sublattice of $L$ containing 0 and $M$ is the lattice of submodules of a distinguished ring $R$. If $D$ has characteristic 0 , then a finite sublattice of $L$ must be distributive in order to be the lattice of submodules of a ring of linear transformations of $M$.

A subspace $N$ of $M$ is called $J$-distributive iff $N \cap(A \cup B)=(N \cap A) \cup(N \cap B)$ for all $A, B \in J$. It is shown that $N$ is $J$-distributive iff $N=M e$ for some idempotent $e \in R$. All subspaces of $M$ are $J$-distributive iff $J$ is a chain. Wolfson proved that $R$ is a Baer ring (i.e., every annihilating right or left ideal of $R$ is generated by an idempotent) if $J$ is a chain. We show that this is almost the only case in which a distinguished ring is a Baer ring.

Every distinguished ring $R$ is a direct sum of subrings of the form $e_{i} R e_{j}$, when $1=e_{1}+\cdots+e_{n}$ (direct sum), each $e_{i} R e_{j}$ is a full ring of linear transformations, $e_{i} R e_{j}=0$ if $i<j$, and $\sum_{i \neq j} e_{i} R e_{j}$ is the radical of $R$. Two distinguished rings are shown to be isomorphic iff their vector spaces are related in an obvious way.

1. Introduction. A module ${ }_{D} M$ over a ring $D$ has associated with it a lattice $L\left({ }_{D} M\right)$ of all submodules and a ring $E\left({ }_{D} M\right)$ of all endomorphisms. For each subring $R$ of $E\left({ }_{D} M\right)$, we shall always consider $M$ to be a bimodule ${ }_{D} M_{R}$. Associated with each sublattice $J$ of $L\left({ }_{D} M\right)$ is a subring $R=R(J)=\left\{r \in E\left({ }_{D} M\right) \mid N r \subset N\right.$ for every $N \in J\}$ of $E\left({ }_{D} M\right)$. Clearly $J \subset L\left(M_{R}\right)$. Let us call a sublattice $J$ of ${ }_{D} M$

Presented to the Society, January 25, 1963; received by the editors February 18, 1963.

(1) This research was supported in part by a grant NSF-G24155. 
a distinguished lattice (d-lattice) of ${ }_{D} M$ iff $J=L\left(M_{R}\right)$. Also, let us call a subring $R$ of $E\left({ }_{D} M\right)$ a distinguished ring (d-ring) iff $R=R(J)$ where $J=L\left(M_{R}\right)$.

The ring $D$ is restricted in this paper to be a division ring and the module ${ }_{D} M$ to be a unital module over $D$ (i.e., a vector space over $D$ ). Thus, every $d$-ring is a ring of linear transformations of some vector space over a division ring. If $C(D)$ denotes the center of $D$, then we may consider $C(D) \subset R(J)$ for every $J \subset L\left({ }_{D} M\right)$ if we define $x a=a x$ for all $a \in C(D)$ and $x \in M$. It is well known that there is associated with each basis $B$ of ${ }_{D} M$ a division ring $D_{B} \subset E\left({ }_{D} M\right)$ isomorphic to $D$. Thus, for each $d \in D$ we define $d^{\prime} \in D_{B}$ by $x d^{\prime}=d x, x \in B$.

It is easily shown that $R\left(L\left({ }_{D} M\right)\right)=C(D)$. Hence, $L\left({ }_{D} M\right)$ is a $d$-lattice of ${ }_{D} M$ iff $D$ is a field. If $B$ is a basis of ${ }_{D} M$ and $J$ is the sublattice of $L\left({ }_{D} M\right)$ generated by all atoms of the form $D x$ and $D(x+y), x, y \in B$, then it can be shown that $R(J)=D_{B}$. On the other hand, if $J$ is generated by only the atoms $D x, x \in B$, then $R(J) \cong \prod_{i \in \Delta} D_{i}$ where $\Delta=\operatorname{card} B$ and $D_{i}=D_{B}$ for each $i \in \Delta$. At the other extreme, $\{0, M\}$ is a lattice of ${ }_{D} M$ having $E\left({ }_{D} M\right)$ as its $d$-ring.

Our primary interest in this paper is with finite $d$-lattices of ${ }_{D} M$ and their associated $d$-rings. Clearly each $d$-lattice of ${ }_{D} M$ is a complete, modular lattice containing 0 and $M$, and $R(J)$ is a ring with unity. For each $N \in J$, we denote the lattice dimension of $N$ by $\operatorname{dim} N(\operatorname{dim} N$ is the length of the longest chain in the interval $[0, N]$ of $J$ ) and let $\operatorname{dim} J=\operatorname{dim} M$. If $J=L\left({ }_{D} M\right)$ then $\operatorname{dim} N$ is the usual vector space dimension of a subspace $N$ of $M$.

If $J$ is any finite-dimensional modular lattice, then $N \in J$ is called irreducible iff $N \neq 0$ and $N$ is not a union of lower-dimensional elements of $J$. Each irreducible $P \in J$ covers a unique element of $J$ which we will always designate by $P^{0}$. Clearly $P^{0}=0$ iff $P$ is an atom of $J$. The set of all irreducible elements of $J$ is designated by $I(J)$. Given a lattice $J$ and $N \in J$, we shall also let $I(N)=\{A \in I(J) \mid A \leqq N\}$.

Given a finite-dimensional distributive lattice $J$, each nonzero $N \in J$ may be uniquely represented as an irreducible union of irreducible elements of $J$, namely as the union of the maximal elements of $I(N)[1$, p. 142]. It is easily verified in this case that $I(A \cup B)=I(A) \cup I(B)$ and $I(A \cap B)=I(A) \cap I(B)$ for all $A, B \in J$. Incidentally, we shall use the notation $\dot{\cup}$ for direct union in a lattice.

The following theorem is of basic importance to the rest of this paper.

1.1 THEOREM. Let $L$ be a complete, complemented, modular lattice with identities 0 and $I$ and $J$ be a finite-dimensional sublattice of $L$ containing 0 and I. For each irreducible $P \in J$, let us select $\bar{P} \in L$ so that $P=\bar{P} \dot{\cup} P^{0}$. Then each nonzero $K \in J$ may be represented in the form $K=\bigcup_{i=1}^{m} \bar{P}_{i}$ for some subset $\left\{P_{1}, \cdots, P_{m}\right\}$ of $I(K)$. If $J$ is distributive, then $K=\bigcup_{i=1}^{n} \bar{P}_{i}$ where $I(K)=\left\{P_{1}, \cdots, P_{n}\right\}$.

Proof. The theorem is trivially true if $\operatorname{dim} K=1$. Let us assume that the conclusion holds for every $K \in J$ of dimension $\leqq n$ and let $K \in J, \operatorname{dim} K=n+1$. If $K$ is irreducible, then $K=K^{0} \dot{\cup}$ and $K$ is a direct union of elements of the 
form $\bar{P}, P$ irreducible, since $K^{0}$ is. If $J$ is distributive, then $I(K)=\{K\} \cup I\left(K^{0}\right)$ so that $K=\bigcup_{P \in I(K)} \bar{P}$ by the inductive assumption.

If $K$ is reducible, then $K=A \cup B$ where $A, B \in J, A$ irreducible, and $\operatorname{dim} B \leqq n$. We know that $A=A^{0} \cup A$ and $A \cap B \leqq A^{0}$. Since $A \cap\left(A^{0} \cup B\right)=A^{0} \cup(A \cap B)=A^{0}$, clearly $A \cap\left(A^{0} \cup B\right) \leqq A \cap A^{0}=0$. Hence, $K=A \dot{\cup}\left(A^{0} \cup B\right)$ and the first conclusion follows from the inductive assumption once we observe that $\operatorname{dim}\left(A^{0} \cup B\right) \leqq n$. If $J$ is distributive, the desired conclusion follows from the observation that $I(K)=\{A\} \cup I\left(A^{0} \cup B\right)$. This proves 1.1 .

2. Distributive lattices of ${ }_{D} M$. Every finite-dimensional distributive lattice is actually finite $\left[1\right.$, p. 139]. A finite, distributive sublattice of $L\left({ }_{D} M\right)$ containing 0 and $M$ will be called a $F D$-lattice of ${ }_{D} M$ henceforth. If $J$ is a $F D$-lattice of ${ }_{D} M$ and $I(J)=\left\{P_{1}, \cdots, P_{n}\right\}$, then by $1.1, M=\bar{P}_{1} \dot{\cup} \cdots \dot{\cup} \bar{P}_{n}$ where $\bar{P}_{i}$ is any relative complement (in $L\left({ }_{D} M\right)$ ) of $P_{i}^{0}$ in $P_{i}, i=1, \cdots, n$.

2.1 TheOREM. If $J$ is a FD-lattice of ${ }_{D} M$ and, for each $P \in I(J), \bar{P} \in L\left({ }_{D} M\right)$ is chosen so that $P=P^{0} \dot{\cup} \bar{P}$, then $R(J)=\left\{a \in E\left({ }_{D} M\right) \mid \bar{P} a \subset P\right.$ for every $\left.P \in I(J)\right\}$.

Proof. If $a \in R(J)$ then clearly $\bar{P} a \subset P a \subset P$ for every $P \in I(J)$. Conversely, if $a \in E\left({ }_{D} M\right)$ and $\bar{P} a \subset P$ for every $P \in I(J)$, then $K=\bigcup_{P \in I(K)} \bar{P}$ for every $K \in J$ and $K a \subset \bigcup_{P \in I(K)} P=K$. Hence, $a \in R(J)$. This proves 2.1 .

It is clear from 2.1 that if $J$ is a $F D$-lattice of ${ }_{D} M, I(J)=\left\{P_{1}, \cdots, P_{n}\right\}$, and $P_{i}=\bar{P}_{i} \dot{\cup} P_{i}^{0}$ for some $\bar{P}_{i} \in L\left({ }_{D} M\right), i=1, \cdots, n$, then for any $a_{i} \in \operatorname{Hom}\left({ }_{D} \bar{P}_{i},{ }_{D} P_{i}\right)$, $i=1, \cdots, n$, there exists a unique $a \in R(J)$ such that $a \mid \bar{P}_{i}=a_{i}, i=1, \cdots, n$. In particular, we have the following result (if we select $\bar{P}_{i}$ to contain each $x_{i}$ ).

2.2 Corollary. If $J$ is a FD-lattice of ${ }_{D} M, I(J)=\left\{P_{1}, \cdots, P_{n}\right\}$, and $x_{i} \in P_{i}$, $x_{i} \notin P_{i}^{0}, i=1, \cdots, n$, then for any $y_{i} \in P_{i}, i=1, \cdots, n$, there exists some $a \in R(J)$ such that $x_{i} a=y_{i}, i=1, \cdots, n$.

2.3 Corollary. If $J$ is a FD-lattice of ${ }_{D} M$, then each $K \in J$ is a cyclic $R(J)$ module.

Proof. If $K=P_{1} \cup \cdots \cup P_{m}$, each $P_{i}$ irreducible, then select $x_{i} \in P_{i}, x_{i} \notin P_{i}^{0}$, $i=1, \cdots, m$, and let $x=x_{1}+\cdots+x_{m}$. If $y \in K$, say $y=y_{1}+\cdots+y_{m}, y_{i} \in P_{i}$, then by $2.2 x_{i} a=y_{i}, i=1, \cdots, m$, for some $a \in R(J)$ and $x a=y$. Hence, $x R(J)=K$ and 2.3 is proved.

\subsection{THEOREM. Every $F D$-lattice of ${ }_{D} M$ is a d-lattice of ${ }_{D} M$.}

Proof. Let $J$ be a $F D$-lattice of ${ }_{D} M$ and $R=R(J)$. To prove that $J$ is a $d$-lattice of ${ }_{D} M$, we need only show that $L\left(M_{R}\right) \subset J$. To this end, let $x \in M, x \neq 0$, $K=x R$, and $N$ be the least element of $J$ containing $x$. Clearly $K \subset N$. Let $\left\{P_{1}, \cdots, P_{m}\right\}$ be the set of maximal elements of $I(N)$, so that $N=P_{1} \cup \cdots \cup P_{m}$, and let $x=x_{1}+\cdots+x_{m}, x_{i} \in P_{i}$. If any $x_{i} \in P_{i}^{0}$, say $x_{1} \in P_{1}^{0}$, then 


$$
x \in P_{1}^{0} \cup P_{2} \cup \cdots \cup P_{m}<N
$$

since $P_{1} \notin P_{1}^{0} \cup P_{2} \cup \cdots \cup P_{m}$. This contradicts the choice of $N$. Hence $x_{i} \notin P_{i}^{0}, i=1, \cdots, m$. It follows as in the proof of 2.3 that $K=x R=N$. Since each cyclic submodule of $L\left(M_{R}\right)$ is contained in $J$ and $J$ is complete, $L\left(M_{R}\right) \subset J$. This proves 2.4 .

If $J$ is a distributive lattice of finite dimension $n>1$, then it is well known that $I(J)$ has $n$ elements and that it may be imbedded in a Boolean algebra $B$ of dimension $n[1, \mathrm{pp} .139,140]$. Given an $n$-dimensional vector space ${ }_{D} M$ and an atomic basis $\left\{A_{1}, \cdots, A_{n}\right\}$ of $L\left({ }_{D} M\right)$, the sublattice $B^{\prime}$ of $L\left({ }_{D} M\right)$ generated by this basis is an $n$-dimensional Boolean algebra. Since $B \cong B^{\prime}, J$ is isomorphic to a sublattice $J^{\prime}$ of $L\left({ }_{D} M\right)$. Clearly $0, M \in J^{\prime}$ and $J^{\prime}$ is a $F D$-lattice of ${ }_{D} M$. This proves the following result.

2.5 THEOREM. Every distributive lattice $J$ of finite dimension $n>1$ is a FDlattice of each $n$-dimensional vector space ${ }_{D} M$.

Not every finite $d$-lattice of a vector space ${ }_{D} M$ need be distributive. For example, if $D$ is a finite field and ${ }_{D} M$ is finite-dimensional, then $L\left({ }_{D} M\right)$ is a finite lattice that is not distributive. However, many finite $d$-lattices of a vector space are distributive according to the next result.

2.6 THEOREM. If the division ring $D$ has characteristic zero, then every finite d-lattice of ${ }_{D} M$ is a FD-lattice of ${ }_{D} M$.

Proof. Let us assume that $J$ is a finite $d$-lattice of ${ }_{D} M$ that is not distributive. Then $J$ must contain a sublattice of the type shown in the figure $[1$, p. 134]. Let

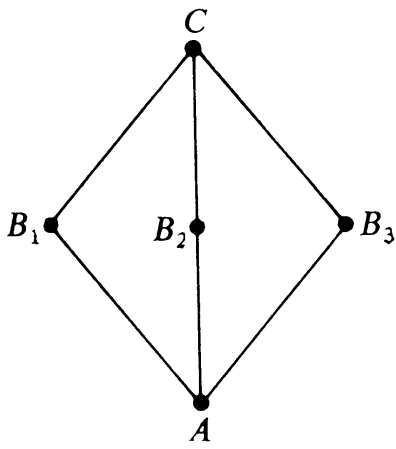

us select $B_{3}^{\prime} \in L\left({ }_{D} M\right)$ such that $B_{3}=A \dot{\cup} B_{3}^{\prime}$ and let $\left\{z_{i} \mid i \in \Delta\right\}$ be a basis of $B_{3}^{\prime}$ in ${ }_{D} M$. Since $B_{3} \subset B_{1}+B_{2}, z_{i}=x_{i}+y_{i}$ for some $x_{i} \in B_{1}$ and $y_{i} \in B_{2}, i \in \Delta$. If $B_{1}^{\prime}$ and $B_{2}^{\prime}$ are the subspaces of $B_{1}$ and $B_{2}$, respectively, generated by $\left\{x_{i} \mid i \in \Delta\right\}$ and $\left\{y_{i} \mid i \in \Delta\right\}$, then $B_{1}=A \cup B_{1}^{\prime}, B_{2}=A \cup B_{2}^{\prime},\left\{x_{i} \mid i \in \Delta\right\}$ is a basis of $B_{1}^{\prime}$, and $\left\{y_{i} \mid i \in \Delta\right\}$ is a basis of $B_{2}^{\prime}$. For if $\Sigma d_{i} x_{i} \in A$ for some $d_{i} \in D$, then $\Sigma d_{i} z_{i} \in B_{2} \cap B_{3}=A, \quad \Sigma d_{i} z_{i}=0$, and each $d_{i}=0$. Hence, $A \cap B_{1}^{\prime}=0$ and $\left\{x_{i} \mid i \in \Delta\right\}$ is a basis of $B_{1}^{\prime}$. Similarly, $A \cap B_{2}^{\prime}=0$ and $\left\{y_{i} \mid i \in \Delta\right\}$ is a basis of $B_{2}^{\prime}$. 
Finally, each $x \in B_{1}$ has the form $x=y+z$ for some $y \in B_{2}$ and $z \in B_{3}$. In turn, $z=z^{\prime}+\sum d_{i} z_{i}$ for some $z^{\prime} \in A$ and $d_{i} \in D$, so that $x=y+z^{\prime}+\sum d_{i} x_{i}+\sum d_{i} y_{i}$. Hence, $x-\sum d_{i} x_{i} \in B_{1} \cap B_{2}=A$. Consequently, $B_{1}=A \dot{\cup} B_{1}^{\prime}$ and, similarly, $B_{2}=A \dot{\cup} B_{2}^{\prime}$.

For each $i \in \Delta$, let us select a nonzero $\gamma_{i}=\left(b_{i}, c_{i}\right) \in C(D) \times C(D)$, and let $B^{\prime}(\gamma)$ be the subspace of ${ }_{D} M$ generated by $\left\{b_{i} x_{i}+c_{i} y_{i} \mid i \in \Delta\right\}$. If $\sum d_{i}\left(b_{i} x_{i}+c_{i} y_{i}\right) \in A$ for some $d_{i} \in D$, then $\sum d_{i} b_{i} x_{i} \in B_{2}$ and $\sum d_{i} c_{i} y_{i} \in B_{1}$. It follows that $\sum d_{i} b_{i} x_{i}$ $=\Sigma d_{i} c_{i} y_{i}=0$ and $d_{i} b_{i}=d_{i} c_{i}=0$ for each $i$. Since $\gamma_{i} \neq 0, d_{i}=0$ for each $i$. We conclude that $B^{\prime}(\gamma) \cap A=0$.

We shall next prove that for each $\gamma=\left\{\gamma_{i} \mid i \in \Delta\right\}$, the subspace $B(\gamma)=A \dot{\cup} B^{\prime}(\gamma)$ of ${ }_{D} M$ is in $J$. First, we observe that $B_{3} a \subset B_{3}$ for each $a \in R=R(J)$, so that $\left(x_{i}+y_{i}\right) a=u_{i}+d_{i}\left(x_{i}+y_{i}\right)$ for some $u_{\imath} \in A$ and $d_{i} \in D, i \in \Delta$. Since $x_{i} a \in B_{1}$ and $y_{i} a \in B_{2}$ for each $a \in R$, we must have $x_{i} a=v_{i}+d_{i} x_{i}$ and $y_{i} a=w_{i}+d_{i} y_{i}$ for some $v_{i}, w_{i} \in A, i \in \Delta$. Hence, $\left(b_{i} x_{i}+c_{i} y_{i}\right) a=b_{i} v_{i}+c_{i} w_{i}+d_{i}\left(b_{i} x_{i}+c_{i} y_{i}\right), i \in \Delta$, and $B(\gamma) a \subset B(\gamma)$ for each $a \in R$. Therefore, $B(\gamma) \in J$.

We easily see that there are an infinite number of distinct subspaces of ${ }_{D} M$ of the form $B(\gamma)$. For example, let $B_{n}^{\prime}$ be the subspace generated by $\left\{(n-2) x_{i}+y_{i} \mid i \in \Delta\right\}$ and let $B_{n}=A \dot{\cup} B_{n}^{\prime}, n=2,3, \cdots$. If $n \neq m$ and $u \in B_{n} \cap B_{m}$, then $u=v+\sum d_{i}\left[(n-2) x_{i}+y_{i}\right]=w+\sum d_{i}^{\prime}\left[(m-2) x_{i}+y_{i}\right]$ for some $v, w \in A$ and $d_{i}, d_{i}^{\prime} \in D, i \in \Delta$. Hence, $\Sigma\left[d_{i}(n-2)-d_{i}^{\prime}(m-2)\right] x_{i}$ $\in B_{1} \cap B_{2}=A$ and, similarly, $\Sigma\left(d_{i}-d_{i}^{\prime}\right) y_{i} \in A$. Consequently,

$$
\Sigma\left[d_{i}(n-2)-d_{i}^{\prime}(m-2)\right] x_{i}=0
$$

and $\Sigma\left(d_{i}-d_{i}^{\prime}\right) y_{i}=0$. Therefore, $d_{i}(n-2)-d_{i}^{\prime}(m-2)=0$ and $d_{i}-d_{i}^{\prime}=0$ for each $i$. From these equations, we easily see that $d_{i}=d_{i}^{\prime}=0$ for each $i$. Hence, $B_{n} \cap B_{m}=A$ for all $m$ and $n, m \neq n$, and $J$ contains the infinite set $\left\{B_{2}, B_{3}, \cdots\right\}$ of subspaces. This is contrary to assumption, and proves 2.6 .

If $J$ is a $F D$-lattice of ${ }_{D} M$, then $J$ has a center $C(J)$ consisting of all complemented elements of $J$. If $\left\{M_{1}, \cdots, M_{k}\right\}$ is the set of atoms of the Boolean algebra $C(J)$ and $J_{i}$ denotes the interval $\left[0, M_{i}\right]$ of $J, i=1, \cdots, k$, then $M=M_{1} \dot{\cup} M_{2} \dot{\cup} \cdots \dot{\cup} M_{k}$ and $J \cong J_{1} \times J_{2} \times \cdots \times J_{k}$. The $d$-rings $R=R(J)$ and $R_{i}=R\left(J_{i}\right), i=1, \cdots, k$, are easily seen to be related by an isomorphism, $R \cong R_{1} \times R_{2} \times \cdots \times R_{k}$, under the correspondence $a \leftrightarrow\left(a_{1}, a_{2}, \cdots, a_{k}\right), a \in R$, where $a_{i}=a \mid M_{i}, i=1, \cdots, k$.

Let us call the $d$-ring $R=R(J)$ indecomposable iff $C(J)=\{0, M\}$. It is evident that $R$ is indecomposable iff $R$ is not a direct union of two nonzero ideals of $R$. For if $R=A \dot{\cup} B, A$ and $B$ nonzero ideals, then $M=M A \dot{\cup} M B$ and $M A$, $M B \in C(J)$. Conversely, if $M=M^{\prime} \dot{\cup} M^{\prime \prime}$ for some nonzero $M^{\prime}, M^{\prime \prime} \in J$ then $R=A \cup \dot{\cup} B$ where $A=\left\{r \in R \mid M r \subset M^{\prime}\right\}$ and $B=\left\{r \in R \mid M r \subset M^{\prime \prime}\right\}$ are nonzero ideals of $R$. Clearly each ring $R_{i}$ above is indecomposable. We state our remarks above in the following form. 
2.7 THEOREM. Every d-ring is isomorphic to a finite direct product of indecomposable d-rings.

3. $J$-distributivity. If $J$ is a $F D$-lattice of ${ }_{D} M$ and $N \in L\left({ }_{D} M\right)$, then $N$ is called $J$-distributive iff $N \cap(A \cup B)=(N \cap A) \cup(N \cap B)$ for all $A, B \in J$. It is easily checked that if $N$ is $J$-distributive and $J_{N}=\{N \cap K \mid K \in J\}$, then $J_{N}$ is a $F D$ lattice of ${ }_{D} N$. Each element of $J$ is $J$-distributive since $J$ is a distributive lattice. It is equally clear that every subspace $N$ of ${ }_{D} M$ is $J$-distributive if $J$ is a chain. A useful characterization of the $J$-distributive elements of $L\left({ }_{D} M\right)$ is given below.

3.1 ThEOREM. If $J$ is a FD-lattice of ${ }_{D} M$ and $N \in L\left({ }_{D} M\right)$, then the following statements are equivalent:

(a) $N$ is $J$-distributive.

(b) For each $P \in I(J)$ there exists $P^{\prime} \in L\left({ }_{D} M\right)$ such that $P^{\prime} \subset P, P^{\prime} \cap P^{0}=0$, and $N=\bigcup_{P \in I(J)} P^{\prime}$.

(c) $N=$ Me for some idempotent $e \in R(J)$.

Proof. Let $N$ be $J$-distributive and for each $P \in I(J)$ let $P^{\prime} \in L\left({ }_{D} M\right)$ be selected so that $\left(N \cap P^{0}\right) \dot{\cup} P^{\prime}=N \cap P$. Clearly $P^{\prime} \cap P^{0}=0$, since

$$
P^{\prime} \cap P^{0} \subset\left(N \cap P^{0}\right) \cap P^{\prime}=0 .
$$

We shall prove that

(1) $N \cap K=\bigcup_{P \in I(K)} P^{\prime}$ for every nonzero $K \in J$.

To prove (1), we note first that it holds if $K$ is an atom of $J$, since then $K^{\prime}=N \cap K$. Let us assume that (1) holds for every element of $J$ of dimension $n$ or smaller, and let $K \in J$ be of dimension $n+1$. Since $N$ is $J$-distributive, $N \cap K=\bigcup_{P \in I(K)} N \cap P=\bigcup_{P \in I(K)}\left[\left(N \cap P^{0}\right) \cup P^{\prime}\right]$. Now $\operatorname{dim} P^{0} \leqq n$ for every $P \in I(K)$, and therefore $N \cap P^{0}=\bigcup_{Q \in I\left(P^{0}\right)} Q^{\prime}$. Hence,

$$
N \cap K=\bigcup_{P \in I(K)}\left[\bigcup_{Q \in I\left(P^{0}\right)} Q^{\prime} \cup P^{\prime}\right]=\bigcup_{P \in I(K)} P^{\prime}
$$

since $I\left(P^{0}\right) \subset I(K)$ for every $P \in I(K)$. This proves (1).

If we let $K=M$ in (1), we obtain (b).

If (b) holds, then for each $P \in I(J)$ we may choose $\bar{P}, P^{\prime \prime} \in L\left({ }_{D} M\right)$ such that $P=P^{0} \cup \bar{P}$ and $\bar{P}=P^{\prime} \dot{\cup} P^{\prime \prime}$. Since $M=\bigcup_{P \in I(J)} \bar{P}$ by Theorem 1.1, there is a well-defined idempotent $e \in E\left({ }_{D} M\right)$ such that $e \mid P^{\prime}=\iota$ and $e \mid P^{\prime \prime}=0$ for each $P \in I(J)$, where $\iota$ designates the identity mapping. Clearly $e \in R(J)$ since $P e \subset P$ for every $P \in I(J)$. Since $N=\bigcup_{P \in I(J)} P^{\prime}, M e=N$ by the very definition of $e$. Thus, (b) implies (c).

Finally, let $e \in R(J)$ be an idempotent and $N=M e$. It is easily shown that $N \cap K=K e$ for every $K \in J$. Hence, $N \cap(A \cup B)=(A+B) e=A e+B e$ $=(N \cap A) \cup(N \cap B)$ for all $A, B \in J$, and $N$ is $J$-distributive. Thus, (c) implies (a). This proves 3.1. 
3.2 Corollary. If $J$ is a FD-lattice of ${ }_{D} M$ and $x \in M, x \neq 0$, then $D x$ is $J$-distributive iff $x \in P, x \notin P^{0}$, for some $P \in I(J)$.

This follows directly from 3.1 , part(b).

For each idempotent $e \in R(J), 1-e$ also is an idempotent in $R(J)$ and $M=M e \dot{\cup} M(1-e)$. By Theorem 3.1, $M e=\bigcup_{P \in I(J)} P^{\prime}$ and $M(1-e)$ $=\bigcup_{P \in I(J)} P^{\prime \prime}$ for some $P^{\prime}, P^{\prime \prime} \in L\left({ }_{D} M\right)$ such that $M e \cap P=\left(M e \cap P^{0}\right) \dot{\cup} P^{\prime}$ and $M(1-e) \cap P=\left[M(1-e) \cap P^{0}\right] \cup P^{\prime \prime}$ for every $P \in I(J)$. It is easy to show that $P=P^{0} \dot{\cup}\left(P^{\prime} \dot{\cup} P^{\prime \prime}\right)$ for each $P \in I(J)$ and hence that $M=\bigcup_{P \in I(J)}\left(P^{\prime}+P^{\prime \prime}\right)$. Since $e \mid P^{\prime}=\iota$ and $e \mid P^{\prime \prime}=0$ for every $P \in I(J)$, we have shown that every idempotent $e \in R(J)$ is arrived at in the way shown in the proof of 3.1.

3.3 THEOREM. If $J$ is a FD-lattice of ${ }_{D} M$ and $S$ is the set of all $J$-distributive elements of $L\left({ }_{D} M\right)$, then $S=L\left({ }_{D} M\right)$ iff $J$ is a chain.

Proof. It is evident that if $J$ is a chain then $S=L\left({ }_{D} M\right)$. If $J$ is not a chain, then there exists a chain $0=K_{0}<K_{1}<\cdots<K_{r}$ contained in $J$ such that (1) if $K \in J$ and $K \neq K_{i}$ for $i=0,1, \cdots, r$, then $K>K_{r}$, and (2) $K_{r}$ is covered by at least two distinct elements $P$ and $P^{\prime}$ of $J$. Evidently $P$, $P^{\prime} \in I(J)$ and $P^{0}=P^{\prime 0}=P \cap P^{\prime}=K_{r}$. Let us select nonzero $x \in P$ and $x^{\prime} \in P^{\prime}$ such that $x, x^{\prime} \notin K_{r}$, and let $N=D\left(x+x^{\prime}\right)$. Clearly $N$ is not $J$-distributive, since $N \cap\left(P \cup P^{\prime}\right)=N$ whereas $N \cap P=N \cap P^{\prime}=0$. This proves 3.3.

4. Projectors. Let $J$ be a $F D$-lattice of ${ }_{D} M$ and $R=R(J)$. For all $K, N \in J$, define the projector of $K$ into $N, K^{-1} N$, to be the largest subset of $R$ such that $K\left(K^{-1} N\right) \subset N$. Clearly $K^{-1} N$ is an ideal of $R$. Since $K R \subset K$, evidently $K^{-1} N=K^{-1}(K \cap N)$. Thus, we might as well assume that $K \supset N$ in discussing the projector of $K$ into $N$. If $A$ is an ideal of $R$ and $N \in J$, then the projector of $A$ into $N, N A^{-1}$, is the largest subset of $M$ such that $\left(N A^{-1}\right) A \subset N$. It is clear that $N A^{-1} \in J$ and $N A^{-1} \supset N$. Finally, if $A$ and $B$ are ideals of $R$, we can define the right and left projectors of $B$ into $A, B^{-1} A$ and $A B^{-1}$, as the largest subsets of $R$ such that $B\left(B^{-1} A\right) \subset A$ and $\left(A B^{-1}\right) B \subset A$, respectively. Evidently $B^{-1} A$ and $A B^{-1}$ are ideals of $R$ containing $A$. Since $A B^{-1}=(A \cap B) B^{-1}$ and $B^{-1} A=B^{-1}(A \cap B)$, we might as well assume that $B \supset A$ in discussing the projectors $B^{-1} A$ and $A B^{-1}$. The properties of projectors we shall need are contained in the following theorem.

4.1 THEOREM. If $J$ is a FD-lattice of ${ }_{D} M$ and $R=R(J)$, then:

(1) $K\left(K^{-1} N\right)=N$ and $N\left(K^{-1} N\right)^{-1}=K$ if $K \supset N, K, N \in J$.

(2) $N_{1}^{-1} N_{2}=\left(N_{1}^{-1} N_{3}\right)\left(N_{2}^{-1} N_{3}\right)^{-1}$ and $N_{2}^{-1} N_{3}=\left(N_{1}^{-1} N_{2}\right)^{-1}\left(N_{1}^{-1} N_{3}\right)$ if $N_{1} \supset N_{2} \supset N_{3}, N_{i} \in J$.

Proof. (1) There exists an idempotent $e \in R$ such that $M e=N$. Hence, $K \cap M e=K e=N, e \in K^{-1} N$, and $K\left(K^{-1} N\right)=N$. To prove the second part, we first observe that $N\left(K^{-1} N\right)^{-1} \supset K$. Let us assume that $N\left(K^{-1} N\right)^{-1}=K^{\prime} \neq K$. 
Then $K^{\prime} \in J$ and there exists some $P \in I\left(K^{\prime}\right)$ such that $P \notin K$. Hence, we can select $P^{\prime} \in L\left({ }_{D} M\right)$ so that $P^{\prime} \dot{\cup} P^{0}=P$. Therefore $P^{\prime} \cap K=0$ and we can select $e \in R$ so that $e \mid K=0$ and $e \mid P^{\prime}=\iota$. Clearly $e \in K^{-1} N$ and $K^{\prime}\left(K^{-1} N\right) \notin N$ since $K^{\prime} e \supset P^{\prime}$. However, $\left[N\left(K^{-1} N\right)^{-1}\right]\left(K^{-1} N\right) \subset N$ according to the definition of $N\left(K^{-1} N\right)^{-1}$. This contradiction proves that $N\left(K^{-1} N\right)^{-1}=K$.

(2) Clearly

$$
\left(N_{1}^{-1} N_{2}\right)\left(N_{1}^{-1} N_{3}\right) \subset N_{1}^{-1} N_{3},
$$

so that $N_{1}^{-1} N_{2} \subset\left(N_{1}^{-1} N_{3}\right)\left(N_{2}^{-1} N_{3}\right)^{-1}$ and $N_{2}^{-1} N_{3} \subset\left(N_{1}^{-1} N_{2}\right)^{-1}\left(N_{1}^{-1} N_{3}\right)$. On the other hand, $\left[\left(N_{1}^{-1} N_{3}\right)\left(N_{2}^{-1} N_{3}\right)^{-1}\right]\left(N_{2}^{-1} N_{3}\right) \subset N_{1}^{-1} N_{3}$ so that $N_{1}\left[\left(N_{1}^{-1} N_{3}\right)\left(N_{2}^{-1} N_{3}\right)^{-1}\right]\left(N_{2}^{-1} N_{3}\right) \subset N_{3}$. Hence, $N_{1}\left[\left(N_{1}^{-1} N_{3}\right)\left(N_{2}^{-1} N_{3}\right)^{-1}\right]$ $\subset N_{3}\left(N_{2}^{-1} N_{3}\right)^{-1}=N_{2}$ and $\left(N_{1}^{-1} N_{3}\right)\left(N_{2}^{-1} N_{3}\right)^{-1} \subset N_{1}^{-1} N_{2}$. Therefore, $\left(N_{1}^{-1} N_{3}\right)\left(N_{2}^{-1} N_{3}\right)^{-1}=N_{1}^{-1} N_{2}$. Similarly, $\left(N_{1}^{-1} N_{2}\right)\left[\left(N_{1}^{-1} N_{2}\right)^{-1}\left(N_{1}^{-1} N_{3}\right)\right]$ $\subset N_{1}^{-1} N_{3}, N_{1}\left(N_{1}^{-1} N_{2}\right)\left[\left(N_{1}^{-1} N_{2}\right)^{-1}\left(N_{1}^{-1} N_{3}\right)\right] \subset N_{3}, N_{2}\left[\left(N_{1}^{-1} N_{2}\right)^{-1}\left(N_{1}^{-1} N_{3}\right)\right]$ $\subset N_{3}$, and $\left(N_{1}^{-1} N_{2}\right)^{-1}\left(N_{1}^{-1} N_{3}\right) \subset N_{2}^{-1} N_{3}$, Therefore, $\left(N_{1}^{-1} N_{2}\right)^{-1}\left(N_{1}^{-1} N_{3}\right)=N_{2}^{-1} N_{3}$, and 4.1 is proved.

If $J$ is a $F D$-lattice of ${ }_{D} M$ and $R=R(J)$, then an ideal of $R$ of the form $A^{-1} 0$, resp. $0 A^{-1}$, for some ideal $A$ of $R$ is called a right, resp. left, annihilating ideal of $R$. Let us designate by $L_{r}(R)$, resp. $L_{l}(R)$, the set of all right, resp. left, annihilating ideals of $R$.

4.2 THEOREM. If $J$ is a FD-lattice of ${ }_{D} M$ and $R=R(J)$, then $L_{r}^{\prime}(R)=\left\{K^{-1} 0 \mid K \in J\right\}$ and $L_{l}(R)=\left\{M^{-1} K \mid K \in J\right\}$. Thus, the mapping $J \stackrel{\alpha}{\rightarrow} L_{r}(R)$ defined by $\alpha K=K^{-1} 0, K \in J$, is a dual isomorphism and the mapping $J \stackrel{\beta}{\rightarrow} L_{l}(R)$ defined by $\beta K=M^{-1} K, K \in J$ is an isomorphism.

Proof. By 4.1, (2), $K^{-1} 0=\left(M^{-1} K\right)^{-1}\left(M^{-1} 0\right)=\left(M^{-1} K\right)^{-1} 0$ and therefore $K^{-1} 0 \in L_{r}(R)$ for each $K \in J$. Similarly, $M^{-1} K=\left(M^{-1} 0\right)\left(K^{-1} 0\right)^{-1}=0\left(K^{-1} 0\right)^{-1}$ and $M^{-1} K \in L_{l}(R)$ for each $K \in J$. If $A \in L_{r}(R)$ and $B=0 A^{-1}$, then $B \in L_{l}(R)$ and $A=B^{-1} 0$. If we let $K=M B$, then $K \in J$ and $K^{-1} 0=B^{-1} 0=A$. Thus, $A$ has the desired form. Also, $M\left(M^{-1} K\right) A=0,\left(M^{-1} K\right) A=0$, and $B \subset M^{-1} K \subset 0 A^{-1}$ $=B$. Hence, $B=M^{-1} K$ and $B$ has the desired form. If $K^{-1} 0=N^{-1} 0$ for some $K, N \in J$, then $K=0\left(K^{-1} 0\right)^{-1}=0\left(N^{-1} 0\right)^{-1}=N$ by 4.1 , (1). If $M^{-1} K=M^{-1} N$ for some $K, N \in J$, then $K=M\left(M^{-1} K\right)=M\left(M^{-1} N\right)=N$ by 4.1 , (1). Thus, $\alpha$ and $\beta$ are bijections. This proves 4.2.

If $J$ is a $F D$-lattice of ${ }_{D} M$ and $R=R(J)$, and if $K, N \in J$ with $K \supset N$, then $K^{-1} N=\left(K^{-1} 0\right)\left(N^{-1} 0\right)^{-1}=\left(M^{-1} K\right)^{-1}\left(M^{-1} N\right)$ by 4.1, (2). That is, $K^{-1} N=(\alpha K)(\alpha N)^{-1}=(\beta K)^{-1}(\beta N)$ if we use the mappings $\alpha$ and $\beta$ of 4.2 . This shows that the ideals of the form $K^{-1} N$, where $K, N \in J$, may be defined intrinsically in the ring $R$ itself. We shall use this fact later on in the paper.

If $J$ is a $F D$-lattice of ${ }_{D} M$ and $K, N \in J$ with $K \supset N$, then evidently $J^{\prime}=\left\{N^{\prime}-N \mid N^{\prime} \in[N, K]\right\}$ is a $F D$-lattice of ${ }_{D}(K-N)$. There is a natural mapping $R \stackrel{\phi}{\rightarrow} R^{\prime}$ of $R=R(J)$ onto $R^{\prime}=R\left(J^{\prime}\right)$ defined by: $(x+N)(c \phi)=x c+N$ 
for all $c \in R$ and $x+N \in K-N$. It is easily seen that $\phi$ is an epimorphism and $\operatorname{ker} \phi=K^{-1} N$. We state this result as follows.

4.3 THEOREM. If $J$ is a FD-lattice of ${ }_{D} M$ and $K, N \in J$ with $K \supset N$, and if $J^{\prime}=\left\{N^{\prime}-N \mid N^{\prime} \in[N, K]\right\}$, then $J^{\prime}$ is a FD-lattice of ${ }_{D}(K-N)$ and $R\left(J^{\prime}\right) \cong R(J) /\left(K^{-1} N\right)$.

5. Baer rings. While we defined the projector $K^{-1} N$ above only for $K, N \in J$, it is clear that $K^{-1} N$ may be defined in the same way for any subsets $K$ and $N$ of $M$ or $R$. In particular, if $J$ is a $F D$-lattice of ${ }_{D} M$ and $R=R(J)$, then we can easily describe $K^{-1} N$ for any $J$-distributive subspaces $K$ and $N$ of $M$. Thus, $K=M e$ and $N=M f$ for some idempotents $e, f \in R$, and $K^{-1} N \supset(1-e) R+R f$. On the other hand, if $a \in R$ then $a \in K^{-1} N$ iff $M e a \subset N$, or iff $e a f=e a$. That is, $a \in K^{-1} N \quad$ iff $a-a f \in(1-e) R$, or iff $a \in(1-e) R+R f$. Hence, $K^{-1} N=(1-e) R+R f$. It follows that $M^{-1} K=R e$ and $K^{-1} 0=(1-e) R$ if $K=M e$. Evidently $M^{-1} K=0\left(K^{-1} 0\right)^{-1}$ and $K^{-1} 0=\left(M^{-1} K\right)^{-1} 0$; that is, $M^{-1} K$ is an annihilating left ideal and $K^{-1} 0$ is an annihilating right ideal of $R$. These are the only annihilating right or left ideals of $R$ generated by idempotents according to the next result.

5.1 THEOREM. Let $J$ be a FD-lattice of ${ }_{D} M$ and $R=R(J)$. If $A$ is a right ideal and $B$ is a left ideal of $R$ such that $A=B^{-1} 0$ and $B=0 A^{-1}$, then $A$ and $B$ are generated by idempotents iff either $0 A^{-1}$ or $M B$ is a J-distributive subspace of $M$.

Proof. If $A=e R$ for some idempotent $e \in R$, then $B=R(1-e)$ and $0 A^{-1}=M B=M(1-e)$. Hence, $0 A^{-1}(=M B)$ is $J$-distributive by 3.1. Conversely, if either $0 A^{-1}$ or $M B$ is $J$-distributive, then either $0 A^{-1}=M f$ or $M B=M e$ for some idempotents $e, f \in R$. If $0 A^{-1}=M f$, then $A \subset(1-f) R$ and $B \subset R f$ since $M B \subset M f$. Hence, $A=B^{-1} 0 \supset(R f)^{-1} 0=(1-f) R \supset A$ and $A=(1-f) R$, $B=R f$. If $M B=M e$, then $(M B)^{-1} 0=B^{-1} 0=(1-e) R=A$ and $B=0 A^{-1}=R e$. This proves 5.1 .

A ring $R$ is called a Baer ring iff every annihilating right or left ideal of $R$ is generated by an idempotent. In a recent paper [2], Wolfson proved the following result.

5.2 THEOREM. If $J$ is a finite chain in $L\left({ }_{D} M\right)$ containing 0 and $M$, then $R(J)$ is a Baer ring.

We point out that this theorem follows directly from 3.3 and 5.1. Thus, for every right ideal $A$ and left ideal $B$ of $R(J)$ such that $A=B^{-1} 0$ and $B=0 A^{-1}$, $M B$ is $J$-distributive by 3.3 and therefore $A$ and $B$ are generated by idempotents according to 5.1 .

It is evident that if the $F D$-lattice $J$ of ${ }_{D} M$ is isomorphic to a direct product of chains, then $R(J)$ is isomorphic to a direct product of Baer rings and hence is a 
Baer ring. However, these are not the only distinguished rings that are Baer rings. We shall not give the details, but it may be shown that the ring $R$ of all $3 \times 3$ matrices over a division ring $D$ of the form $d_{11} e_{11}+d_{22} e_{22}+d_{31} e_{31}+d_{32} e_{32}$ $+d_{33} e_{33}$, where $e_{i j}$ are the usual matrix units, is a Baer ring. In this case, we may also represent $R$ as $R(J)$, where $J=\left\{0, D x_{1}, D x_{2}, D x_{1}+D x_{2}, M\right\}$ and $M=D x+D x_{2}+D x_{3}$, a 3-dimensional vector space over $D$. If the lattice $J$ is slightly more complicated, then $R(J)$ is not a Baer ring as the following theorem shows.

5.3 THEOREM. If the FD-lattice $J$ of ${ }_{D} M$ contains three irreducible elements $P_{1}, P_{2}, P_{3}$ such that $P_{1} \cup P_{2} \subset P_{3}$ and $P_{1} \cap P_{2}$ is different from $0, P_{1}$, and $P_{2}$, then $R=R(J)$ is not a Baer ring.

Proof. Let $\left\{P_{1}, \cdots, P_{n}\right\}=I(J)$ and let $P_{i}^{\prime} \in L\left({ }_{D} M\right)$ be selected so that $P_{i}^{0} \cup P_{i}^{\prime}=P_{i}, i=1, \cdots, n$. Select $x_{i} \in P_{i}^{\prime}, x_{i} \notin P_{i}^{0}, i=1,2,3$, and let $N=D\left(x_{1}+x_{2}\right)$. Since $N \cap\left(P_{1} \cup P_{2}\right)=N$ whereas $\left(N \cap P_{1}\right) \cup\left(N \cap P_{2}\right)=0, N$ is not $J$-distributive. If we imagine that $x_{3} \in B_{3}$, a basis of $P_{3}^{\prime}$, then there exists $b \in R$ such that $x_{3} b=x_{1}+x_{2}, x b=0$ for $x \in B_{3}, x \neq x_{3}, P_{i}^{\prime} b=0$ if $i \neq 3$. Clearly $M b=N$, and therefore $N^{-1} 0=b^{-1} 0=A$ is an annihilating right ideal of $R$. If $A$ were generated by an idempotent, then $K=0 A^{-1}$ would be a $J$-distributive subspace of $M, K \supset N$. Now for any nonzero $y \in P_{1} \cap P_{2}$, there exists some $a \in R$ such that $x_{1} a=y, x_{2} a=-y$, and $a \mid P_{i}^{\prime}=\imath$ for $i=3, \cdots, n$. Since $\left(x_{1}+x_{2}\right) a=0$, evidently $a \in A$. However, $P_{i} a \neq 0$ for $i=1, \cdots, n$, and therefore $P_{i} \notin K, i=1, \cdots, n$. It follows that $K=0$, contrary to the fact that $K \supset N$. Consequently, $A$ is not generated by an idempotent. This proves 5.3.

For example, if $M=D x_{1}+D x_{2}+D x_{3}+D x_{4}$ is a 4-dimensional vector space over $D$ and $J=\left\{0, D x_{1}, D x_{1}+D x_{2}, D x_{1}+D x_{3}, D x_{1}+D x_{2}+D x_{3}, M\right\}$, then $R=R(J)$ is the ring of all $4 \times 4$ matrices over $D$ of the form

$$
\left[\begin{array}{llll}
a_{11} & 0 & 0 & 0 \\
a_{21} & a_{22} & 0 & 0 \\
a_{31} & 0 & a_{33} & 0 \\
a_{41} & a_{42} & a_{43} & a_{44}
\end{array}\right] .
$$

In the notations of 5.3, $P_{1}=D x_{1}+D x_{2}, P_{2}=D x_{1}+D x_{3}$, and $P_{3}=M$. Letting $N=D\left(x_{2}+x_{3}\right)$ as we did in the proof of 5.3, $N^{-1} 0=A=e_{11} R+\left(e_{21}-e_{31}\right) R+e_{44} R$ and $B=0 A^{-1}=R\left(e_{42}+e_{43}\right)$. Since $B^{2}=0$, evidently $B$, and therefore also $A$, is not generated by an idempotent.

6. Subrings of distinguished rings. Let $J$ be a $F D$-lattice of ${ }_{D} M$ and $R=R(J)$. An idempotent $e \in R$ is called simple iff $e \neq 0$ and there exists an irreducible $P \in J$ such that $M e \subset P$ and $M e \cap P^{0}=0$. A sum $e=e_{1}+\cdots+e_{m}$ of idempotents 
$e_{i} \in R$ is called direct iff $e_{i} e_{j}=\delta_{i j} e_{j}, i, j=1, \cdots, m$. Evidently a direct sum of nonzero idempotents of $R$ is a nonzero idempotent of $R$.

We have seen that if $\left\{P_{1}, \cdots, P_{n}\right\}=I(J)$, then associated with each nonzero idempotent $e \in R$ is a splitting $P_{i}=P_{i}^{0} \dot{\cup} P_{i}^{\prime} \dot{\cup} P_{i}^{\prime \prime}, i=1, \cdots, n$, such that $M e=\bigcup_{i=1}^{n} P_{i}^{\prime}$ and $M(1-e)=\bigcup_{i=1}^{n} P_{i}^{\prime \prime}$. If we define $e_{i} \in R$ as follows: $e_{i} \mid P_{i}^{\prime}=\imath$, $e_{i}\left|P_{j}^{\prime}=e_{i}\right| P_{k}^{\prime \prime}=0, i \neq j, i, j, k=1, \cdots, n$, then clearly $e_{i} e_{j}=\delta_{i j} e_{j}, i, j=1, \cdots, n$, and $e=e_{1}+\cdots+e_{n}$. Since each nonzero $e_{i}$ is simple, we have proved the following result.

6.1 THEOREM. If $J$ is a FD-lattice of ${ }_{D} M$, then each nonzero idempotent in $R(J)$ is a direct sum of simple idempotents.

If $e=e_{1}+\cdots+e_{m}$ is a direct sum of simple idempotents of $R=R(J)$, then the subring $e R e$ of $R$ is a direct sum of the subrings $e_{i} R e_{j}$. Let us now describe the structure of each subring $e_{i} R e_{j}$.

6.2 THEOREM. If $J$ is a FD-lattice of ${ }_{D} M$ and $e, f$ are nonzero idempotents of $R=R(J)$, then $e R f \cong S=\left\{a \in \operatorname{Hom}\left({ }_{D}(M e),{ }_{D}(M f)\right) \mid(K e) a \subset K f\right.$ for every $K \in J\}$.

Proof. We can consider $S \subset E\left({ }_{D} M\right)$ by letting $a \mid M(1-e)=0$ for each $a \in S$. Since $(K e) e R f \subset K f$ for each $K \in J$, evidently $e R f \subset S$. Now $K S=[K e+K(1-e)] S=(K e) S \subset K f \subset K$ for each $K \in J$, and therefore $S \subset R$. Since $x a=x e a f$ for all $x \in M$ and $a \in S, S=e S f \subset e R f$. Thus, $S=e R f$ and 6.2 is proved.

6.3 COROLLARY. If $J$ is a FD-lattice of ${ }_{D} M$, $e$ is a nonzero idempotent of $R=R(J)$, and $N=$ Me, then $e R l \cong R\left(J_{N}\right)$.

We recall from $\S 3$ that $J_{N}=\{N \cap K \mid K \in J\}$. It follows from 6.3 that every subring of a $d$-ring $R$ of the form $e R e, e$ an idempotent, is also a $d$-ring.

6.4 THEOREM. Let $J$ be a FD-lattice of ${ }_{D} M$ and e, $f$ be simple idempotents of $R=R(J)$ such that $M e \subset P, M e \cap P^{0}=0$, and $M f \subset Q, M f \cap Q^{0}=0$, for given irreducible $P, Q \in J$. Then $e R f \neq 0$ iff $Q \subset P$. If $e R f \neq 0$, then $e R f \cong$ $\operatorname{Hom}\left({ }_{D}(M e),{ }_{D}(M f)\right)$.

Proof. If $Q \subset P$ then $M e R f=P f \neq 0$ and $e R f \neq 0$. If $Q \notin P$ then $P f \subset M f \cap Q \cap P \subset M f \cap Q^{0}=0, M e R f=0$, and $e R f=0$. In case $Q \subset P$ and $K \in J$, we have $K e \subset K \cap P \neq 0$ iff $K \supset P$. Hence, $(K e) a \subset K f$ for all $a \in \operatorname{Hom}\left({ }_{D}(M e),{ }_{D}(M f)\right)$ and $K \in J$, and $e R f \cong \operatorname{Hom}\left({ }_{D}(M e),{ }_{D}(M f)\right)$ in view of 6.2. This proves 6.4 .

Let us call ring $R$ a full ring iff $R=E\left({ }_{D} N\right)$ for some vector space ${ }_{D} N$. If $J$ is a $F D$-lattice of ${ }_{D} M$ and $R=R(J)$, then $R$ is a full ring iff $J=\{0, M\}$. Hence, the result below follows directly from 6.3 . 
6.5 THEOREM. If $J$ is a FD-lattice of ${ }_{D} M$ and $e$ is a nonzero idempotent of $R=R(J)$, then $e R e$ is a full ring iff $e$ is simple.

6.6 COROLLARY. The ring eRe of 6.5 is a division ring iff $\operatorname{dim}(M e)=1$ in $L\left({ }_{D} M\right)$. If $e R e$ is a division ring, then $e R e \cong D$.

That $e R e \cong D$ is seen as follows. Since $\operatorname{dim}(M e)=1, M e=D x$ for some $x \in M e$. Now $x(e R e) \in L\left({ }_{D} M\right)$ and therefore $x(e R e)=D x$. Hence, the mapping $e R e^{\sigma} \rightarrow D$ defined by: xere $=(\text { ere })^{\sigma} x, r \in R$, is an isomorphism.

If $J$ is a $F D$-lattice of ${ }_{D} M$ and $\left\{P_{1}, \cdots, P_{n}\right\}=I(J)$, then we can order this set so that $P_{j} \notin P_{i}$ if $j>i, i, j=1, \cdots, n$ (for example, order $I(J)$ so that $\operatorname{dim}\left(P_{i}\right) \leqq \operatorname{dim}\left(P_{i+1}\right)$ in $\left.J\right)$. Let $1=e_{1}+\cdots+e_{n}$ be a direct sum of simple idempotents of $R=R(J)$, with $M e_{i} \cup P_{i}^{0}=P_{i}, i=1, \cdots, n$. Then $e_{i} R e_{j}=0$ if $j>i$ and $R=\sum_{j \leqq i=1}^{n} e_{i} R e_{j}$. Thinking in terms of a matrix representation of $R$, this says that the representation can be broken up into $n^{2}$ blocks with the blocks down the main diagonal full matrix rings and the blocks above the main diagonal all zero. The subring

$$
A=\sum_{j<i=1}^{n} e_{i} R e_{j}
$$

clearly is an ideal of $R$. Since $A^{n}=0$ and $R / A \cong e_{1} R e_{1}+\cdots+e_{n} R e_{n}$, a direct sum of primitive rings, evidently $A$ is the Jacobson radical of $R$. We have proved part of the following theorem.

6.7 THEOREM. Let $J$ be a FD-lattice of ${ }_{D} M$ of dimension $n$ and $R=R(J)$. Then 1 can be expressed as a direct sum of $n$ simple idempotents of $R, 1=e_{1}+\cdots+e_{n}$, in such a way that $R$ is a direct sum of subrings

The subring

$$
R=\sum_{j \leqq i=1}^{n} e_{i} R e_{j}
$$

$$
A=\sum_{j<i=1}^{n} e_{i} R e_{j}
$$

is the Jacobson radical of $R$, and

$$
R / A \cong e_{1} R e_{1}+\cdots+e_{n} R e_{n},
$$

a direct sum of full rings. The ideal $A$ is nilpotent with index of nilpotency one less than the length of the longest chain in $I(J)$.

Proof. We need only find the index of nilpotency of $A$ to complete the proof. Let $I(J)=\left\{P_{1}, \cdots, P_{n}\right\}$, with $\operatorname{dim} P_{i} \leqq \operatorname{dim} P_{i+1}, i=1, \cdots, n-1$. By 6.4, $e_{i} R e_{j} \neq 0$ iff $P_{i} \supset P_{j}$; and if $P_{i} \supset P_{j}$ then $e_{i} R e_{j} \cong \operatorname{Hom}\left({ }_{D}\left(M e_{i}\right),{ }_{D}\left(M e_{j}\right)\right)$. If $e_{i} R e_{j} \neq 0$ and $e_{j} R e_{k} \neq 0$, then for every nonzero $a \in e_{i} R e_{j}$ there exists some $b \in e_{j} R e_{k}$ such that $a b \neq 0$. It follows that $A^{m-1} \neq 0$ iff there exists a chain 
$P_{i_{1}} \supset P_{i_{2}} \supset \cdots \supset P_{i_{m}}$ in $I(J)$. Hence, the index of nilpotency of $A$ is the length of the longest chain in $I(J)$.

7. Isomorphic theorems. Distinguished rings are isomorphic iff the obvious isomorphisms hold between their underlying vector spaces, as we shall show below. Our results are extensions and generalizations of those of Wolfson [2, Theorem 7].

7.1 THEOREM. Let $J$ and $J^{\prime}$ be FD-lattices of ${ }_{D} M$ and ${ }_{D^{\prime}} M^{\prime}$, respectively, and let $R=R(J)$ and $R^{\prime}=R\left(J^{\prime}\right)$. Then $R \cong R^{\prime}$ iff there exist isomorphisms $D \stackrel{\sigma}{\rightarrow} D^{\prime}$ and $J \stackrel{\alpha}{\rightarrow} J^{\prime}$ such that $\operatorname{dim}_{D}\left(P-P^{0}\right)=\operatorname{dim}_{D^{\prime}}\left(\alpha P-\alpha P^{0}\right)$ for every $P \in I(J)$.

Proof. Let $R_{\stackrel{\phi}{\rightarrow}}^{\rightarrow} R^{\prime}$ be an isomorphism. Then the mapping $J \stackrel{\alpha}{\rightarrow} J^{\prime}$ defined by: $\alpha K=M^{\prime}\left[\left(M^{-1} K\right) \phi\right], K \in J$, is an isomorphism by 4.2. If $P \in I(J)$ and $P^{\prime}=\alpha P$, then $E\left({ }_{D}\left(P-P^{0}\right)\right) \cong R /\left(P^{-1} P^{0}\right)$ and $E\left({ }_{D^{\prime}}\left(P^{\prime}-P^{\prime 0}\right)\right) \cong R^{\prime} /\left(P^{\prime-1} P^{\prime 0}\right)$ by 4.3 . Since $\left(P^{-1} P^{0}\right) \phi=\left[\left(M^{-1} P\right)^{-1}\left(M^{-1} P^{0}\right)\right] \phi=\left(M^{\prime-1} P^{\prime}\right)^{-1}\left(M^{\prime-1} P^{\prime 0}\right)=\left(P^{\prime-1} P^{\prime 0}\right)$, we conclude that $E\left({ }_{D}\left(P-P^{0}\right)\right) \cong E\left({ }_{D^{\prime}}\left(P^{\prime}-P^{\prime 0}\right)\right)$ for every $P \in I(J)$. By a classical result of ring theory $[3$, p. 79$]$, we have that $D \cong D^{\prime}$ and $\operatorname{dim}_{D}\left(P-P^{0}\right)$ $=\operatorname{dim}_{D^{\prime}}\left(P^{\prime}-P^{\prime 0}\right)$ for every $P \in I(J)$.

Conversely, let us assume that $D^{\boldsymbol{\sigma}} \rightarrow D^{\prime}$ and $J \stackrel{\alpha}{\rightarrow} J^{\prime}$ are isomorphisms such that $\operatorname{dim}_{D}\left(P_{i}-P_{i}^{0}\right)=\operatorname{dim}_{D^{\prime}}\left(\alpha P_{i}-\alpha P_{i}^{0}\right), i=1, \cdots, n$, where $I(J)=\left\{P_{1}, \cdots, P_{n}\right\}$. We may select $\bar{P}_{i} \in L\left({ }_{D} M\right)$ and $\bar{P}_{i}^{\prime} \in L\left({ }_{D}, M^{\prime}\right)$ such that $P_{i}=\bar{P}_{i} \dot{\cup} P_{i}^{0}$ and $\alpha P_{i}=P_{i}^{\prime}=\bar{P}_{i}^{\prime} \dot{\cup} P_{i}^{\prime 0}, i=1, \cdots, n$. By assumption, there exist bases $\left\{x_{i j} \mid j \in \Delta_{i}\right\}$ of $\bar{P}_{i}$ and $\left\{x_{i j}^{\prime} \mid j \in \Delta_{i}\right\}$ of $\bar{P}_{i}^{\prime}, i=1, \cdots, n$. Each element of $M$ is uniquely represented as a finite sum of the form $\Sigma_{i, j} d_{i j} x_{i j}, d_{i j} \in D$, by 1.1. Thus, there is a unique mapping $M \stackrel{\theta}{\rightarrow} M^{\prime}$ defined by: $\left(\sum d_{i j} x_{i j}\right) \theta=\Sigma d_{i j}^{\sigma} x_{i j}^{\prime}$. It is clear that $\theta$ is a $1-1$ semilinear mapping of $M$ onto $M^{\prime}$ that maps $J$ onto $J^{\prime}$. Hence the mapping $E\left({ }_{D} M\right) \stackrel{\psi}{\rightarrow} E\left({ }_{D} M^{\prime}\right)$ defined by: $a \psi=\theta^{-1} a \theta, a \in E\left({ }_{D} M\right)$, is an isomorphism [3, p. 45]. If $r \in R$, so that $P_{i} r \subset P_{i}, i=1, \cdots, n$, then $P_{i}^{\prime}(r \psi)=P_{i}^{\prime}\left(\theta^{-1} r \theta\right)=P_{i}(r \theta) \subset P_{i} \theta \subset P_{i}^{\prime}, i=1, \cdots, n$, and $r \psi \in R^{\prime}$. Conversely, if $r \psi \in R^{\prime}$ then $P_{i}^{\prime}(r \psi)=P_{i}(r \theta) \subset P_{i}^{\prime}, P_{i} r \subset P_{i}^{\prime} \theta^{-1}=P_{i}, i=1, \cdots, n$, and $r \in R$. Thus, if we let $\phi=\psi \mid R$, the mapping $R \stackrel{\phi}{\rightarrow} R^{\prime}$ is an isomorphism. This proves 7.1.

\section{BIBLIOGRAPHY}

1. G. Birkhoff, Lattice theory, Amer. Math. Soc. Colloq. Publ. Vol. 25, rev. ed., Amer. Math. Soc., Providence, R.I., 1948.

2. K. G. Wolfson, Baer subrings of the ring of linear transformations, Math. Z. 75 (1961), 328-332.

3. N. Jacobson, Structure of rings, Amer. Math. Soc. Colloq. Publ. Vol. 37, Amer. Math. Soc., Providence, R. I., 1956.

4. E. A. Behrens, Distributiv darstellbare Ringe, Math. Z. 73 (1960), 409-432; II, Math. Z. 76 (1961), 367-384.

UNIVERSITY OF ROCHESTER, ROCHESTER, NEW YORK 